\title{
Indirect searches for Dark Matter with the ANTARES neutrino telescope
}

\author{
Guillaume LAMBARD* on behalf of the ANTARES Collaboration \\ IFIC-Instituto de Fisica Corpuscular, Edificio Institutos de Investigacion, Apdo. de Correos \\ 22085, E-46071 Valencia - Spain \\ E-mail: guillaume.lambardeific.uv.es
}

\begin{abstract}
Using the ANTARES neutrino telescope, the largest neutrino telescope in the Northern hemisphere, with its first configuration with 5 lines of photodetectors to the actual nominal one corresponding to a total of 12 lines, we have studied our ability to search indirectly for an evidence of Dark Matter annihilations in heavy astrophysical objects as the Sun. First results have been obtained using the data recorded by ANTARES in 2007 and 2008, and compared with neutrino fluxes predicted within a minimal supersymmetric extension of the Standard Model with supersymmetry-breaking scalar and gaugino masses constrained to be universal at the GUT scale, the CMSSM, as well as a minimal Universal Extra-Dimensions scenario with one extra compact dimension where all the Standard Model fields propagate into the bulk, the UED. The current limits over the neutrino/muon fluxes coming from Dark Matter annihilations, and the spin-dependent cross-section with protons, as well as the expected sensitivities predicted after several years of data taking with ANTARES will be presented.
\end{abstract}

XXIst International Europhysics Conference on High Energy Physics

21-27 July 2011

Grenoble, Rhônes Alpes France

\footnotetext{
* Speaker.
} 


\section{Introduction}

As the very old problem about the unseen planets, measuring a deviation from the known laws of gravitation and the theory of general relativity in large astrophysical systems, we assume the existence of a huge amount of unseen matter, the dark matter [1], which represents $83 \%$ of the matter in the universe. Massives, and weakly interacting with the matter, the WIMPs (Weakly Interacting Massive Particles), defining the dark matter nature whatever a particular framework in Particle Physics, can be elastically scattered in a medium to be decelerated, and gravitationally attracted in heavy objects as the galactic center, or the stars like our Sun which is the point source of the present analysis. It's through the self-annihilation of WIMPs into the Sun's core, that muon neutrinos (independently of electron, and tau neutrinos) can be produced in an energy range reachable to a neutrino telescope as ANTARES.

Since the 29th of May 2008, the neutrino telescope ANTARES has been completed at $40 \mathrm{~km}$ offshore from Toulon at $2475 \mathrm{~m}$ depth, making it the largest neutrino telescope in the northern hemisphere, and the first to operate in the deep sea [2]. It is made up of 12 mooring detection lines holding ten-inch photomultiplier tubes, distributed into 25 storeys on each line, designed for the measurement of neutrino induced charged particles based on the detection of Čerenkov light emitted in water. With a low energy threshold of about $20 \mathrm{GeV}$ for well reconstructed muons, and an angular resolution of about $1^{\circ}$ at low energy $\left(E_{v}<1 \mathrm{TeV}\right)$, ANTARES has a great potential to detect neutrinos induced by the self-annihilation of WIMPs into the Sun's core.

\section{Dark Matter signal, and efficiency of ANTARES}

Model-independently, the differential spectrum of neutrinos at the surface of the Earth, as a function of the mass of the WIMP $M_{\text {WIMP }}$, have been computed using the software package WIMPSIM [3] for all possible self-annihilation channels which can be reached in the Sun's core $(q \bar{q}, l \bar{l}$, $\left.\phi \phi^{*}, v \bar{v}\right)$ in the two common frameworks studied: CMSSM, and mUED. All of the physical contributions have been taken into account as the three-flavors oscillations, or the $\tau$ lepton regeneration through the Sun medium. An averaged effective area $A_{\text {eff }}\left(M_{W I M P}\right)$ for the ANTARES telescope, with the Sun as a point source, is defined as a function of the WIMP masses, a $Q$ track fit quality cut based on a fast reconstruction algorithm [5], and a $\Psi$ half-cone angle around the source, over the neutrino energy range $E_{V} \in\left[10 \mathrm{GeV} ; M_{W I M P}\right]$, weighted by the normalized differential amount of neutrinos for the $W^{+} W^{-}, b \bar{b}, \tau \bar{\tau}$ channels, and for the mUED merged channels. These $A_{e f f}\left(M_{W I M P}\right)$ have been computed for each $(Q, \Psi)$, and for a set of thirteen different WIMP chosen masses in a range [10,1000] GeV (see [17] for details).

\section{Results and discussions}

Following the Model Rejection Factor (MRF) technique [6] to optimize the $(Q, \Psi)$ cuts couple in the Sun direction, the sensitivity in neutrinos $\bar{\phi}_{v}^{90 \%}$ of ANTARES must be maximized for each value of WIMP mass, and each considered channel in the dark matter self-annihilation. For this, $\bar{\phi}_{v}^{90 \%}$ can be expressed as: 

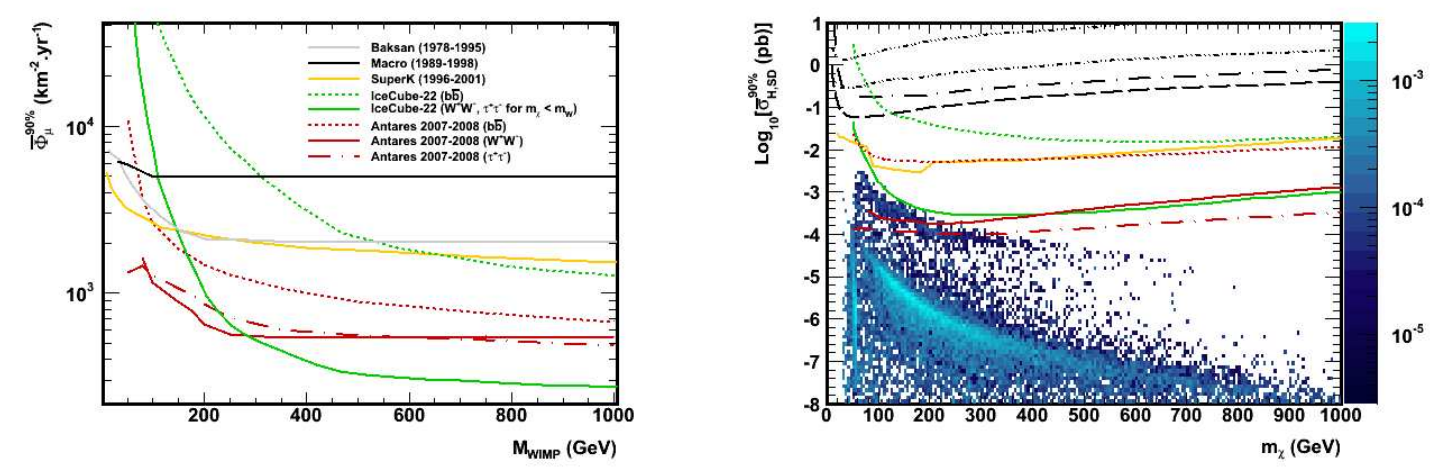

Figure 1: Sentivity $\bar{\phi}_{v}^{90 \%}$ in muon flux as a function of the WIMP masses, in the range $M_{W I M P} /$ in $[10 \mathrm{GeV} ; 1$ $\mathrm{TeV}$ ] and from the data 2007-2008, for the CMSSM framework, on the left side, with Baksan 1978 - 1995 (in grey), Macro 1989 - 1998 (in black), SuperKamiokande 1996 - 2001 (in yellow), IceCube-22 for the $b \bar{b}$ channel in 104 days (in green dashed line), IceCube-22 for the $W^{+} W^{-}$channel in 104 days (in green solid line), ANTARES 2007 - 2008 for the $b \bar{b}$ channel (in red dashed line), ANTARES $2007-2008$ for the $W^{+} W^{-}$channel (in red solid line), and ANTARES 2007-2008 for the $\tau \bar{\tau}$ channel (in red dot-dashed line). And the right side, the spin-dependent cross-section $\bar{\sigma}_{H, S D}^{90 \%}$ as a function of the WIMP masses, in the range $M_{W I M P} /$ in $[10 \mathrm{GeV} ; 1 \mathrm{TeV}]$ and from the data 2007-2008, for the CMSSM framework, with a comparison to the present direct detection experiments as KIMS 2007 [12] (dash-dot black line), CDMS 2010 [11] (dash-two-dots black line), COUPP 2011 [13] (dashed black line), or Picasso 2009 [14] (dashthree-dots black line), and the indirect experiments like IceCube-22 [15] (dashed green line for $b \bar{b}$, solid green line for $W^{+} W^{-}$), SuperKamiokande 1996 - 2001 [16] (yellow solid line). The ANTARES 2007 2008 contributions appear for the $b \bar{b}$ channel (dashed red line), for the $W_{+} W_{-}$channel (solid red line), and for the $\tau \bar{\tau}$ channel (red dot-dashed line).

$$
\bar{\phi}_{v}^{90 \%}=\frac{\bar{\mu}^{90 \%}}{A_{e f f}\left(M_{W I M P}\right) \times T_{e f f}},
$$

where $\bar{\mu}^{90 \%}$ is the average upper limit considering a Poisson statistics in the Feldman-Cousins approach [7], which has been computed from a scrambled data set extracted from the 2007-2008 data taking period of ANTARES, where the configuration of the detector has evolved from a 5 detection lines structure to the final one at 12 lines. Then, $T_{\text {eff }} \simeq 292.9$ days is the effective time of 2007-2008 data taking period, and $A_{\text {eff }}\left(M_{\text {WIMP }}\right)$ is the effective area defined in 2. In order to compare properly the sensitivity in neutrino flux from ANTARES 2007-2008 data to the other experiments, the sensitivity in muon flux must be produced, using the cross section of neutrinos with the Earth medium, the muon range, the nucleon density in the vicinity of the detector, and the neutrino transmission probability through the Earth, all derived from the atmospheric neutrinos (anti-neutrinos) Monte-Carlo. Fig. 1 (left) shows it for the CMSSM framework, as an example. The mUED equivalent can be find in [17].

All of these sensitivities, for both frameworks studied, can be compared to the theoretical parameter spaces allowed by the experimental constraints, and derived from the SuperBayes simulation for the CMSSM framework [8], and for mUED [9]. In this way, the spin-dependent crosssection with protons $\bar{\sigma}_{H, S D}^{90 \%}$ is developped from the sensitivity $\bar{\phi}_{\mu}^{90 \%}$ using the method described in 
[10], as it appears in fig. 1, for the CMSSM (right). For CMSSM and mUED, more the theoretical parameter space has a clear color more this one is allowed by the actual experimental contraints, with a comparison to the present indirect and direct detection experiments. These curves show a $b \bar{b}$ channel sensitivity very closed to the SuperKamiokande one, and better than the IceCube-22 one for the same channel with more than one order of magnitude for the low energy regime $\left(m_{\chi} \leq 180\right.$ $\mathrm{GeV}$ ), and a $W^{+} W^{-}$channel sensitivity better than the IceCube-22 one until $m_{\chi} \sim 440 \mathrm{GeV}$, to be very closed to this last one for higher $m_{\chi}$ mass values. Concerning the $\tau \bar{\tau}$ channel, no equivalent has been found for IceCube or others, which places ANTARES as the actual best one for this channel in the CMSSM framework. This fact can be explained by the energy threshold of ANTARES around $20 \mathrm{GeV}$, lower than for IceCube $\sim 50 \mathrm{GeV}$, which has an effect over all the WIMP mass range due to the integration over the neutrino energy range, $E_{v} \in\left[10 \mathrm{GeV} ; M_{W I M P}\right]$.

\section{Acknowledgments}

We thank the support of the spanish MICINN's Consolider-Ingenio 2010 Programme under grant MultiDark CSD2009-00064.

\section{References}

[1] G. Bertone, D. Hooper, J. Silk, Phys.Rept., 2005, 405: pp. 279-390

[2] J.A. Aguilar et al., NIM in Physics Research, 2011, A 656: pp. 11-38

[3] J. Edsjö, http://www.physto.se/ edsjo/wimpsim/

[4] D. Hooper, S. Profumo, Phys. Rept., 2007, 453, 29

[5] J. Brünner, Astropart. Phys., 2011, 34: pp. 652-662

[6] G.C. Hill, K. Rawlins, Astropart. Phys., 2003, 19: pp. 393-402

[7] G.J. Feldman, R.D. Cousins, Phys. Rev., 1998, D 57: pp. 3873-3889

[8] R. Trotta et al., JHEP 0812:024, 2008

[9] G. Bertone et al., Phys. Rev., 2011, D83, 0306008

[10] G. Wikström, J. Edsjö, 2009, JCAP04, 009

[11] Z. Ahmed et al., Phys. Rev. Lett., 2009, 102, 011301

[12] H. S. Lee et al., Phys. Rev. Lett., 2007, 99, 091301

[13] E. Behnke et al., Science, 2008, 319, 933

[14] S. Archambault et al., Phys.Lett. B, 2009, 682: pp. 185-192

[15] C. de los Heros et al., Proceeding of IDM 2010, Montpellier, France

[16] S. Desai et al., Phys. Rev., 2004, D70, 083523

[17] Proceedings to ICRC2011 by ANTARES collaboration, arXiv:1112.0478 